\title{
ADVANCED AIRFOIL FABRICATION
}

\author{
James R. Dobbs and Jeffrey A. Graves \\ General Electric Company \\ Corporate Research and Development \\ Schenectady, NY 12301 \\ Sergey Meshkov \\ Rybinsk Motor-Building Design Bureau \\ Rybinsk, Yaroslavl Region, RUSSIA
}

\begin{abstract}
Improved turbine engine performance can be obtained by incorporating advanced materials which can tolerate higher turbine inlet temperatures. Higher inlet temperature in itself does not guarantee improved performance if a concomitant increase in cooling air is required. Such parasitic losses would reduce efficiency and can actually result in a net decrease in overall performance. Therefore the greatest likelihood for achieving a significant performance boost or durability increment will be obtained through a coordinated program that strives to improve both airfoil material temperature capability while maintaining or improving cooling efficiency.
\end{abstract}

This paper describes a manufacturing process under development by General Electric which addresses these issues. In addition, Rybinsk Motor-Building Bureau, Russia, has been independently pursuing a similar technology and has been instrumental in demonstrating proof of concept of several key technological issues.

Under development is a fabrication scheme for producing advanced turbine airfoils which will allow the use of novel materials and the flexibility of tailoring the microstructures while maintaining or improving cooling efficiency. In addition, these goals are targeted to be met at a cost which is comparable to current high performance airfoils. The process involves using electron beam physical vapor deposition for applying an outer skin on an inner spar made of conventionally cast single crystal Ni-base superalloy or an intermetallic.

\section{$\underline{\text { Introduction }}$}

\section{Background}

During the past 30 years turbine airfoil temperature capability has increased on average by about $4^{\circ} \mathrm{F}$ per year. Two major factors which have made this increase possible are 1) advanced processing techniques which either improved alloy cleanliness (which leads to improved properties) or enabled the production of tailored microstructures such as directionally solidified or single crystal material, and 2) alloy development resulting in higher use-temperature materials primarily through the addition of substantial additions of refractory elements such $\mathrm{Re}, \mathrm{W}, \mathrm{Ta}$, and Mo.

Although the incipient melting temperature of nickel alloys has been steadily increased (thus contributing to a higher use-temperature), eventually, as the incipient melting temperature approaches the melting point of the nickel alloys, the increased performance benefits versus the cost of developing and processing the alloys undergoes diminishing returns ${ }^{1}$. Superalloy advocates rightfully point out that predictions of $\mathrm{Ni}$-base superalloys' imminent demise due to use-temperature requirements approaching the alloys' theoretical temperature capability have been voiced for many years. During this time $\mathrm{Ni}$ alloys remained the dominate material in the hot section of modern turbine engines and continued to post increases in temperature capability. In spite of this robustness, it must be acknowledged that inevitably the limit of nickel alloys will indeed be reached, at which point avenues other than alloying and process refinement must be sufficiently mature for transition into a production setting.

Current state-of-the-art superalloy turbine blade surface temperatures are near $1150^{\circ} \mathrm{C}\left(2100^{\circ} \mathrm{F}\right)$ while the most severe combinations of stress and temperature corresponds to an average bulk metal temperature approaching $1000^{\circ} \mathrm{C}\left(1830^{\circ} \mathrm{F}\right)^{2}$. Recent in-house studies at GE have documented several "double wall" concepts that provide $15-25 \%$ reductions in cooling airfoil requirements without a rise in surface temperature. Unfortunately, such airfoil concepts are extremely difficult to produce in directional castings because of the small dimensions and fragility of the ceramic core. Blade cost can be extremely high when the casting procedure results in a poor yield of successful cores. However, it may be possible to produce double-walled airfoils economically by 
an unconventional process using electron beam physical vapor deposition (EB-PVD) ${ }^{3}$ for applying an outer skin on a single crystal Ni-base superalloy or intermetallic spar as the interior wall.

\section{Spar-Skin Concept}

The conceptual airfoil shown in Figure 1 has a central spar of a single crystal superalloy with a relatively simple inner cooling plenum geometry. This simplicity allows a substantial increase, as much as $25-33 \%$, in the directional solidification casting yield as compared to current complex single crystal castings.

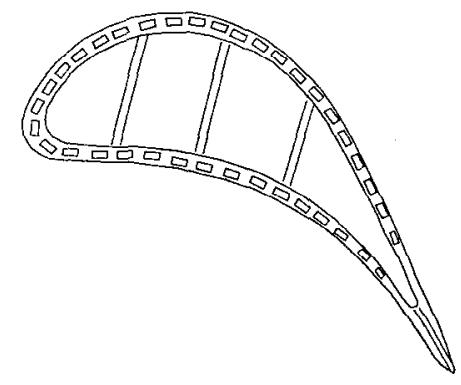

Figure 1 Conceptual Hollow Airfoil Designed for Affordable Fabrication

A general step-by-step procedure for the formation of the spar/skin blade is shown in Figure 2. In brief, the process, which is being termed Optimal Manufacturing for Efficiency Gains in Airfoils (OMEGA), involves casting the inner spar, machining cooling passages on the spar surface, filling the grooves with a sacrificial filler, depositing an outer skin, and leaching out the filler. The savings from decreased casting costs can offset the added cost for producing the airfoil outer skin configuration. Part of the added cost is for forming channels approximately $0.1 \mathrm{~cm}\left(0.040^{\prime \prime}\right)$ deep by $0.255 \mathrm{~cm}(0.1 ")$ wide on the outer surface of the spar casting that eventually serve as the cooling channels between the double walls. As envisioned, the channels will be cast directly in the spar wall, although conventional machining, waterjet machining, ECM, or photoengraving techniques for forming the channels are alternative low-cost processes being evaluated. Cooling channels produced in these ways are very accurately positioned with respect to the wall, so metal currently added to castings to account for core shifts can be eliminated, producing a lighter structure. Dimensions of the cooling channel can be made to the designer's needs, rather than being dominated by the fragility of ceramic core materials thus dramatically improving design flexibility for the cooling channels.

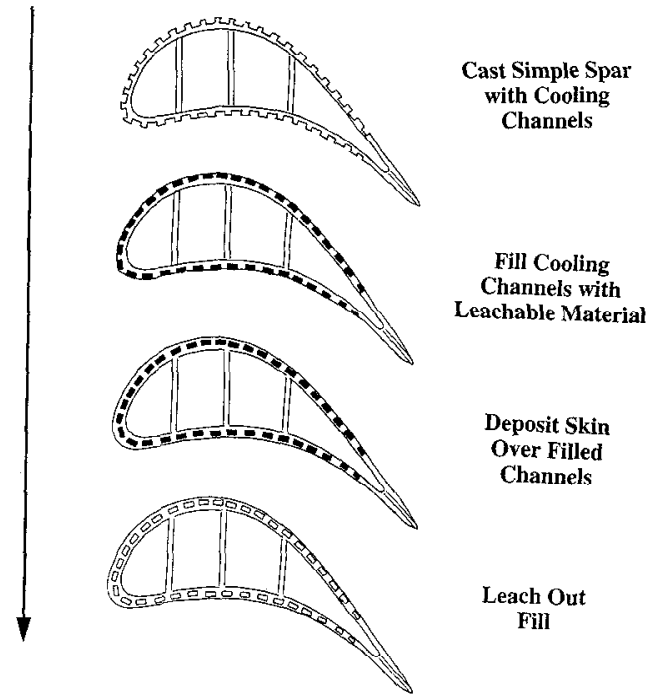

Figure 2 Overview of processing steps for fabrication of spar-skin turbine airfoil.

The spar and outer skin can be either a monolithic superalloy or an intermetallic/superalloy laminate. The strengthening phase may be a silicide intermetallic (e.g. $\mathrm{Cr}_{3} \mathrm{Si}$ ), a topologically close-packed phase such as $\sigma$, or a compound (e.g. $\mathrm{TiC})^{2}$. Intermetallic laminate composites as the skin can operate at temperatures higher than conventional Ni-base single crystal superalloys, and the projected specific strengths of the superalloy-toughened intermetallic matrix composites (IMCs) far exceed those of Ni-base superalloys at the maximum operating temperatures of most turbine airfoils. The temperature capability gain and possible weight reduction due to specific strength improvement can be augmented by the more efficient cooling schemes possible from the skin deposition process. One attractive feature of the spar/skin concept is that its implementation can range from a blade with a simple cooling geometry using a conventional superalloy spar and skin to an advanced blade with complex cooling and an intermetallic spar and skin which is not attainable by current casting techniques. This flexibility allows for an evolutionary approach to integrating advanced materials and advanced cooling concepts.

\section{Development Strategy and Status}

The fundamental strategy adopted for developing this processing scheme addresses the following key points: 1) demonstration of lab-scale feasibility of individual processing procedures including spar casting, channel filling, and skin deposition, 2) estimation of cost differential compared to exiting fabrication schemes, 3 ) analytical predictions of performance gains or durability improvements using the OMEGA process and incorporating existing as well as new blade designs, 4) 
spar/skin concept validation, and 5) rig or engine demonstration.

The major programmatic accomplishment to-date has been the design and fabrication of the EB-PVD unit. While cost analysis and initial performance modelling results have also been completed, the bulk of the material development and characterization work still lies ahead. What follows is a brief description of progress made in each of the development areas.

\section{1) Lab-Scale Feasibility}

One of the attractive features of the OMEGA process is that most of the processing steps consist of fairly conventional techniques. In particular, the casting requirements for the single crystal spar are less demanding than for blades which are currently in commercial production since there is no need for small, fragile, and complex cores to create the cooling channels. Obviously as the complexity of the OMEGA blade increases so do the design challenges. In one scenario both the spar and skin will be IMC laminates, at which point the techniques for casting the IMC spar will need to be developed. However in the near-term version of the OMEGA blade, casting of the Ni-base single crystal spar with the cooling channels should not require additional development. If casting of the surface grooves proves to be impractical, machining of these surface grooves on the cast spar can be accomplished with standard techniques.

Basic feasibility was addressed by EDM'ing $1 / 2$ "-O.D., 3/8" I.D. tubes from $\mathrm{N} 4$ single crystals with eight $0.060^{\prime \prime}$ diamcter scmi-circular grooves machincd in a symmetric pattern in the surface. 60-mil diameter Kovar tubes were placed in the channels to act as a filler and the assembly coated with Rene 142 by plasma spraying. After skin deposition the Kovar was etched out in an acid bath. The samples were then subjected to 100 cycles in a combustion burner rig. The cycles consisted of a 1-hour hold in the flame at $1800^{\circ} \mathrm{F}$ followed by a 5-minute hold in blowing room air. The cooling air was passed up the I.D. and out impingement holes at the top of the specimen then down the cooling channels in a single circuit. After completion of the test, metallography indicated no cracking or distress in either the spar or skin had occurred. While neither the use of Kovar tubes as the filler or the use of plasma spraying as the technique of applying the skin are optimal, this first test did demonstrate basic feasibility.

Channel Filling: The technological issue to be resolved in connection with the cooling channels is to identify a filler material that can completely fill the machined channels while retaining its dimensional stability after curing and during the subsequent skin deposition.
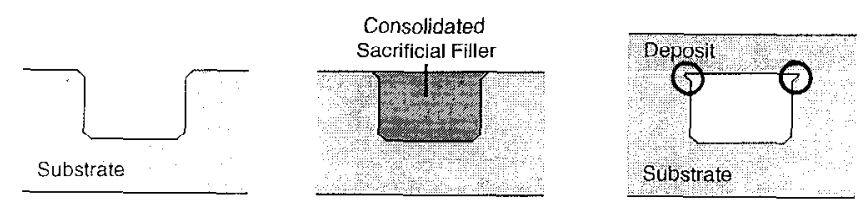

Figure 3 Sketch of channel filling and skin deposition. Care must be taken when designing the surface groove geometry to avoid creating stress concentration sites.

Depending on the method chosen for skin deposition, the filler material must be able to withstand temperatures of up to $1000^{\circ} \mathrm{C}\left(1830^{\circ} \mathrm{F}\right)$ during processing. Such temperatures are proposed to ensure large grains are formed in the deposited skin, thus providing adequate creep resistance. In addition, once the skin has been deposited the filler must be capable of being completely dissolved from the small passages. Potential materials under consideration include ceramics, metals, and ceramic-metal mixtures. Some filler consolidation processes being examined are cold-isostatic pressing (CIP) plus sintering, injection plus sintering, hot isostatic pressing (HIP), and CIP plus electroconsolidation.

Thus far no ideal material has been identified. Ceramics tend to have poor adhesion and a low coefficient of thermal expansion (CTE). The low CTE leads to separation from the channel wall during skin deposition. Metal filler adhesion depends on the consolidation process. In particular, pressureless sinters lead to poor adhesion while the pressure-assisted densification routes give good adhesion. In addition to identifying a filler material, the remaining obstacles include finding a reproducible method of exactly filling the channels so that machining is not required to obtain a flat surface. Also, design of the deposit-skin interfacc is an issue to insure that no stress intensification occurs which may initiate cracks (Figure 3 ).

Skin Deposition: The issues concerning skin deposition center around the desired architecture of the skin. The greatest performance gains will be achieved by using hightemperature intermetallics as the spar and skin material. As an evolutionary first step toward this goal, current work also includes efforts on single crystal superalloy spars (produced by conventional casting techniques) and polycrystalline superalloy skins applied by either EB-PVD or plasma. Although not possessing higher temperature capabilities than current materials, a superalloy spar/skin blade will benefit from reduced cooling requirements due to the advanced cooling passage geometries and thus greater engine efficiencies, while at the same time having similar manufacturing costs (as detailed in the next 
section) as conventional blades. While EB-PVD will allow the more complex architectures to be deposited, reproducible compositional control of materials composed of a wide range of vapor pressure constituents has not been achieved. For that reason plasma sprayed skins are being examined as a near-term alternative while the mechanics of the EB-PVD system are refined. Once the spar/skin concept has been validated using superalloys, the more advanced spar and skin laminated materials will be pursued. It is at this stage that the use of EB-PVD would be required since successive layers of alternate materials can be deposited in a multi-pool unit by controlling which melt pool is being vaporized. Such material layering would not be feasible in a plasma system.

In the following sections, the progress and issues arising in each of these deposition techniques are outlined.

EB-PVD: The EB-PVD process heats and evaporates a molten pool with the condensale collected on a substrate near the source. It is a high-rate process since local temperatures in the liquid pool near the electron beam source may be as high as $4400^{\circ} \mathrm{C}\left(7950^{\circ} \mathrm{F}\right)$. The molten pool is supplied with fresh material either from an adjacent liquid or solid source or from a bottom-fed rod stock.

The rate of metal deposition by EB-PVD may be as high as $25 \mu \mathrm{m}(1 \mathrm{mil})$ per minute. This is two orders of magnitude faster than the fastest rates of sputter deposition processes, and it is this feature that brings the cost of EB-PVD processing of high-temperature components within the range of current aircraft engine blade commercial manufacturing costs. On the other hand, while pure elements and multi-component alloys with similar vapor pressures are fairly easy to evaporate, alloys which contain elements with vastly different vapor pressures are more difficult because vapor concentrations over the pool and the resultant condensate are much less prcdictablc. In addition, small fluctuations in pool depth, solid feed rate, or pool temperature may cause major fluctuations in the vapor concentration over time.

A major milestone was achieved in mid-1994 when an advanced manufacturing-scale evaporator, dedicated to metallic development processes became operational at GE-CKD. A sketch of the unit is shown in Figure 4. The $300 \mathrm{Kw}$ unit is capable of computer-controlled rotation and oscillation of substrates over two separate evaporation zones each of which can have three pools. The controlled oscillation and rotation will permit control of thickness on complex airfoil shapes, as well as the application of microlaminated structures. The advantage of using multiple pools is that elements with low vapor pressures (such as $\mathrm{Mo}, \mathrm{Ta}, \mathrm{Nb}$, and $\mathrm{Ti}$ ) can be isolated and melted independent of the higher vapor pressure elemental constituents. With this arrangement, processing parameters such as ingot feed rate and electron beam power can be tailored to the individual pools and thereby optimize the deposition rate while closely controlling chemistry. In addition, there are two $8 \mathrm{KW}$, electronbeam over-source substrate heaters for accurate substrate temperature control. Access for thermocouple or IR substrate temperature measurement is also provided. A Mikron Corporation Model 9004 Imaging Radiometer is used to measure the pool temperatures and uniformity of heating. The data will be used in an on-going modelling program for understanding both the molten metal pool dynamics and for design of advanced crucibles. Evaporation rates of several kilograms per hour have been achieved with collection rates exceeding $25 \mu \mathrm{m} / \mathrm{min}$. Oxygen content in deposits was found to be less than 50 ppm due to a unique vacuum load chamber and seal system.

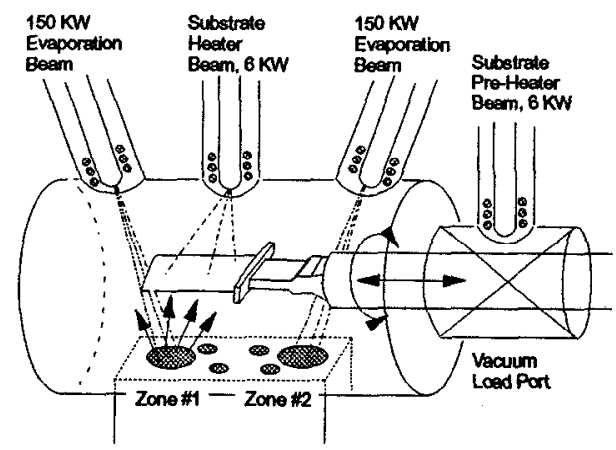

Figure 4 Sketch of EB-PVD unit showing multiple ingot feed pools, dual evaporation beams, and substrate heaters.

As a further aid to compositional control, melting is being performed using the "through-tungsten" approach. In this technique, tungsten is incorporated in the melt pool not as an constituent in the deposit but as a molten pool stabilizer. During melting, the tungsten, because of its extremely low vapor pressure, permeates the melt and acts to regulate the volatilization of all the elements in the pool to some predictable, uniform rate. This results in both greater deposition rates and a more stable composition.

While correcting the ubiquitous mechanical problems which inevitably arise in custom-built equipment, work is progressing on perfecting the multiple pool and through$\mathrm{W}$ approach so as to allow for the deposition of superalloy compositions within tight compositional tolerances. The first trials have used a single pool to deposit a relatively simple target alloy chemistry of 71-Ni, 10.6-Cr, 13.2-Al, 5.3-Co (at.\%). 40-mil thick deposits have been made and are currently being analyzed for chemical uniformity both in the as-deposited and homogenized condition. Once this composition can be deposited, more complex alloys will be attempted. As the alloy compositions approach those of commercial superalloys, 
development of multi-pool depositing will be required to handle the wide range of vapor pressures present.

Plasma Spraying: Plasma spray processes utilize the energy contained in a thermally ionized gas to melt partially and propel fine powder particles on to a surface such that they adhere and agglomerate to produce a coating. The principal advantage of plasma its is ability to deposit complex compositions reliably. In theory, any material that melts without dissociating or subliming can be sprayed using a plasma arc.

Plasma deposits were performed using the RF-plasma spray facility at GECRD. Because of the superior heating characteristics of RF compared to DC plasma, the RF process allows the use of larger size powder for spraying. The resulting larger grain size and lower oxygen content, together with the cleaner plasma environment, yields mechanical properties which are superior to those possible with DC plasma. This is based on GECRD's experience ${ }^{4}$ with Rene 80 where yield stress was found to be comparable to cast material and no severe ductility loss was noted. As for creep resistance, the RF plasma material had ruptures lives at $1500^{\circ} \mathrm{F}$ of $120 \mathrm{hrs}$ on average compared to $1000 \mathrm{hrs}$ for the cast material. It is obvious that even with the larger grain sizes possible in RF plasma, improved creep resistance remains the critical concern for plasma-deposited material.

It is acknowledged that plasma spraying is not capable of producing intermetallic laminate skin structures, and in the as-sprayed condition the long-term creep and stress rupture properties may be insufficient for the demands placed on turbine blades. Inclusion of plasma material in the current program is justified in that it does provide a convenient means of applying superalloy skins on spars and thus allows for an initial evaluation of the spar/skin concept while the EB-PVD technique matures. It is expected that many of the most important issues which could eventually arise from the plasma work will be present in the EB-PVD material as well. Some of these issues which are likely to be common to skins applied by both plasma and PVD are skin adhesion, the effect of a skin material on the fatigue properties of the single crystal spar, thermal expansion mismatch effects, and electrochemical differences. Some issues which are more pertinent to plasma skins are porosity and grain size (since plasma is restricted to monolithics and can't rely on laminates for creep resistance).

The Ni-base alloys chosen for the developmental work are single crystal Rene N5 for the spar material and Rene 142 as the skin. Rene 142 is the directionally solidified version of N5 with the addition of grain boundary strengtheners. A complete characterization of the plasma-deposited Rene 142 material has been a first priority. Material attributes of interest include basic tensile properties, heat treatment, porosity, chemistry, etc. These properties must be determined because published data on Rene 142 is available primarily for material in the directionally solidified condition. Since the plasma sprayed skin has no crystallographic texture and may be somewhat different compositionally, it is crucial that the material properties in the sprayed condition be documented.

The first attempts at depositing Rene 142 were unexpected in that unacceptably high porosity levels $(\sim 7 \%)$ were noted. Numerous additional runs were performed with different processing conditions but to no avail; porosity levels remained at about the $7 \%$ level. It has become necessary to perform a full design of experiments (DOE) matrix in order to find the optimum conditions to achieve as-sprayed low-porosity material. As of this writing the DOE results were not available.

Another study is underway to find a heat treatment schedule that can be applied to a Rene N5/Rene 142 composite spar/skin component. Although compositionally similar, the heat treatment timetemperature profile for the Rene N5 and 142 are significantly different (due primarily to the presence of the grain boundary strengtheners in Rene 142). This means that a modified heat treatment must be developed which can be applied to the composite structure without degrading the properties of either constituent.

Finally, Rene 142 coatings have been applied to N5 single crystals to characterize the interfacial stability and strength as well as the fatigue, creep, and stress rupture properties of the "composite".

When the PVD process matures to the point where complex compositions can be deposited reliably, the knowledge-base derived from the plasma studies can be transferred to the EB-PVD material. In addition to the experience gained in the plasma studies it is possible that the plasma sprayed spar/skin material may be a viable structure in its own right if some of the issues such as small grain size can be controlled or accounted for in the design of the skin.

\section{2) Cost}

One of the first program priorities was to generate a detailed flowchart of the envisioned manufacturing procedure. From this, potential suppliers were consulted in order to develop a realistic cost assessment to ensure that the process was economically feasible. Costs were evaluated at the line item level. The basis of the cost comparison was an aero airfoil design approximately equivalent to a typical fighter engine first stage blade. The external airfoil shape was maintained and a cooling cavity design possible via the Omega process was substituted. Heat transfer and cooling effectiveness of the new cooling cavities were not determined, but were conservatively estimated. Actual cavity design would probably be less rigorous and subsequently less costly. Based on these 
parameters, initial Cost estimates for the Omega process showed that highly cooled multi-walled airfoils could be produced for approximately $70 \%$ of the cost of a similar investment casting.

In addition to initial costs of producing airfoils, there may be additional savings in terms of life-cycle costs of IMC blades. Used single crystal blades cannot presently be refurbished to original properties; tip cracks above the tip cap can be repaired only to equiaxed properties, but cracks below the cap are not repairable. For IMC-skinned airfoils, machining or etching the skin back to the plane of the single crystal spar may allow spar recovery through re-deposition. This would allow a significant increase in repairability and thus spar life.

\section{4) Spar/Skin Concept Demonstration}

The Rybinsk Motors Building Design Bureau (RKBM) of Rybinsk, Russia has provided a successful demonstration of the shell-spar cooling design concept for the production of gas turbine airfoils (Figure 5). Such components have
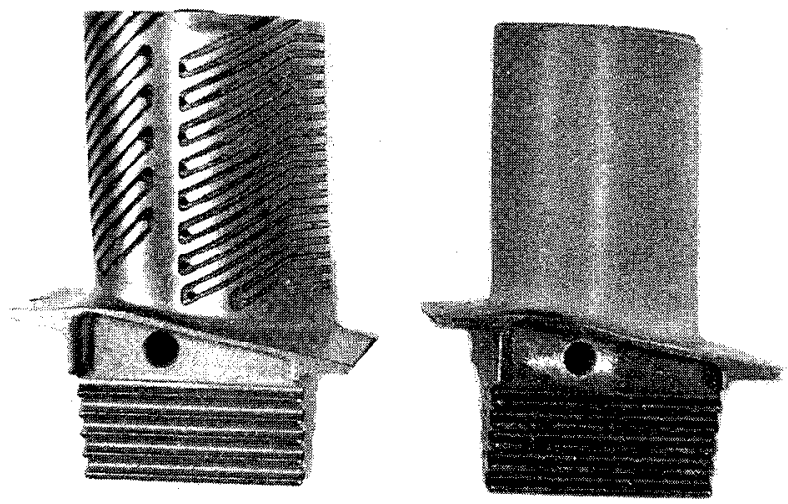

Figure 5 D277 engine turbine blade. On the left is the spar casting and on the right the finished blade after skin deposition.

been designed and used in both aerospace and industrial gas turbine engines, including the D277 turbofan engine and RD-38 lift engine. Current engine experience has been extremely successful, exceeding 1000 hours in Rybinsk aerospace gas turbine applications. RKBM has achieved these accomplishments as a result of over ten years of development experience using investment cast spars with Mcraly skins applied using a modified Movchan EB-PVD coaters. In addition to its significant experience with the production of highly cooled turbine airfoil components, RKBM also has extended this concept to the production of internally heated compressor blades for the TVD-1500 engine, to impart anti-icing capabilities.

Using the shell-spar technology RKBM is able to increase the cooling effectiveness of the turbine blades above that provided by combined convective and film cooling schemes used by present production turbine blade designs (Figure 6). Such cooling effectiveness is believed to be

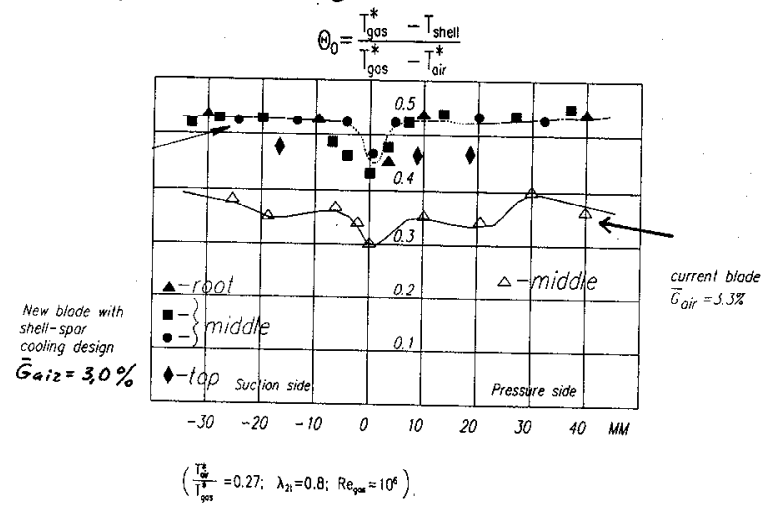

Figure 6 Experimental results comparing cooling effectiveness of current blades (open symbols) to shell-spar blades (filled symbols) on RD-38 engine.

similar to that provided using transpiration cooled airfoils $6,7,8$. In addition, the technology enables designers to reduce temperature gradients present within the cooled airfoils by optimization of the cooling hydraulics. Using this attribute it is possible to maximize the components durability to thermal-mechanical fatigue distress. A third advantage of the concept is the inherent flexibility it provides for rapid optimization of the airfoil cooling design. By machining in the external cooling passages of the hollow spar, various cooling designs may be rapidly and inexpensive produced for rig testing prior to committing to a final optimized configuration for investment casting. It is anticipated that this characteristic of the technology can reduce the design cost of cooled airfoil components. In addition, the increased cooling effectiveness enables the use of low cost superalloys and casting processes to offset the inherently high manufacturing cost of the EB-PVD airfoil skin.

To-date, engine tests of shell-spar components have been conducted using Mcraly airfoil skins. The Rybinsk modifications to the Movchan EB-PVD coater permit the rapid deposition of a multi-layer airfoil skin in which the chemical composition of the skin is gradually varied to produce controlled thermal compatibility with the underlying superalloy spar (Figure 7).

Mechanical verification of the shell-spar structure is obtained using internally cooled hollow shell-spar test specimens to which cyclic mechanical loads are applied both longitudinal and transverse to the channel orientation. When combined with the component structural analysis, the fatigue strength of the relatively weak Mcraly skin was verified to resist the components anticipated thermal and mechanical fatigue loading. Such procedures are also effective for development and evaluation of new spar-skin material systems. 


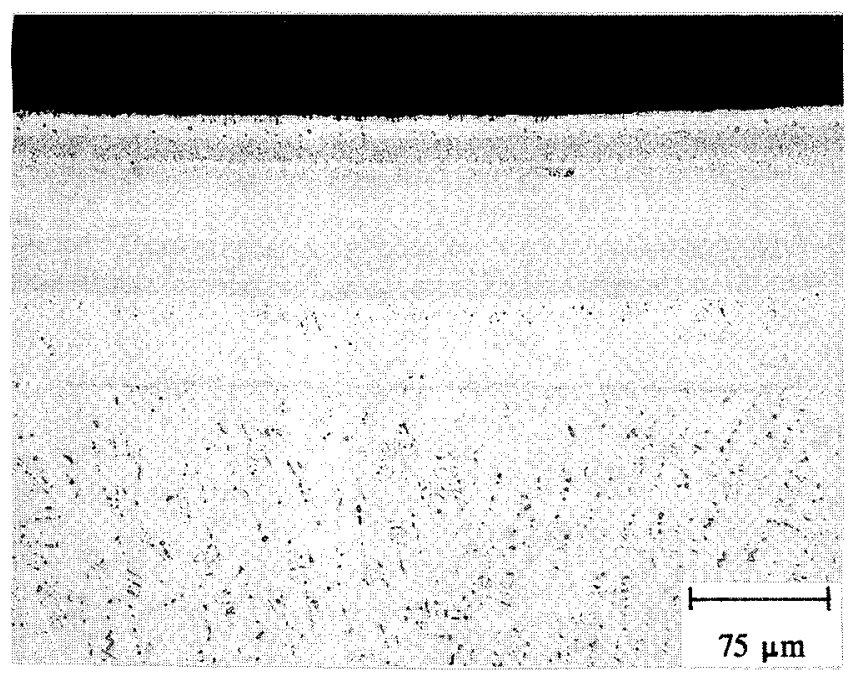

Figure 7 Multi-layered MCrAlY skin used on D277 turbine blade. Skin composition is gradually modified to match thermal compatibility between the superalloy spar and the environmentally protective external MCrAlY coating.

With the recent discovery of their mutual interest in shellspar technology, RKBM has recently joined the ongoing efforts of GE shell-spar program and plans are being made to accelerate the introduction of this new manufacturing technology in the turbine airfoil supplier base. The organizations pursuing this airfoil concept have a common goal of development, optimization, and widespread availability of the shell-spar airfoil technology within the international gas turbine industry.

\section{5) Engine Demonstrator}

The first introduction of the OMEGA process in a General Electric engine is scheduled to occur in 1997. Utilizing the OMEGA process, leading-edge, rear-frame struts for the XTC-76 IHPTET demonstrator will be manufactured by EB-PVD with a $\mathrm{NiCoCrAl}$-base alloy with refractory-element additions as the skin on a conventionally cast N5 spar. For this first application, the fill material is envisioned to be a simple Monel, which previous experiments have shown can be fully etched from the small cooling passages. It is anticipated that this demonstrator will provide valuable engine-environment information for guiding future development of this technology.

\section{Summary}

A proposed process for fabricating advanced cooling geometry turbine airfoils with tailored microstructures by electron beam physical vapor deposition has been described. The major accomplishments of the program thus far are 1) completion of the EB-PVD unit, 2) deposition of simple $\mathrm{NiCoCrAl}$ alloys and Rene 142 on N4 and N5 single crystals, 3) successful completion of burner-rig testing of $\mathrm{R} 142 / \mathrm{N} 4$ without distress to spar or skin, and 4) predicted economic feasibility and performance benefits from the new procedure. Additionally, with the recent teaming of Rybinsk MotorBuilding Design Bureau, development of this technology could be greatly accelerated because of their experience in both design and implementation of the spar-skin concept amassed over the past decade.

\section{References}

1. F.O. Soechting, "A Design Perspective on Thermal Barrier Coatings", in Thermal Barrier Coating Workshop, NASA Conference Proceedings, CP 3312, Cleveland, OH, March 27-29, 1995, pp. 1-15.

2. M.R. Jackson, B.P. Bewley, R.G. Rowe, D.W. Skelly, and H.A. Lipsitt: "High-Temperature Refractory Metal-Intermetallic Composites", JOM, Vol. 48, No. 1, pp. 39-44.

3. D.H. Boone, "Physical Vapour Deposition Process", Mat. Sci. Tech., Vol. 2, 1986, pp. 220-204.

4. P.A. Siemers, M.R. Jackson, J.R. Rairden, and S.D. Savkar: "Rapid Solidification Plasma Deposition (RSPD) for Fabrication of Advanced Aircraft Gas Turbine Components - Volume II", AFWAL-TR86-4071, 1986.

5. S. Ashley, Mech. Eng., 1995, pp. 66-69.

6. H. Mueller-Largent and D.J. Frasier, Final Report NAPC-PE-225-C, 1991.

7. P.S. Burkholder, M.C. Thomas, D.J. Frasier, J.R. Whetstone, K. Harris, G.L. Erickson, S.L. Sikkenga, and J.M. Eridon, Institute of Materials 3rd International Charles Parsons Turbine Conference, Materials Engineering in Turbines and Compressors, Newcastle Upon Tyne, U.K., (April 25th-27th 1995).

8. Fullagar K.P.L., Broomfield R.W., Hulands M., Harris K., Erikson G.L., Sikkenga S.L., 39th ASME/IGTI International Gas Turbine \& Aero Engine Congress \& Expo, The Hague Netherlands, June 13th-16th, 1994). 